\title{
A NOTE ON THE COUNTABLE UNION OF PRIME SUBMODULES
}

\author{
M. R. POURNAKI and M. TOUSI
}

(Received 11 November 2000)

\begin{abstract}
Let $M$ be a finitely-generated module over a Noetherian ring $R$. Suppose $\mathfrak{a}$ is an ideal of $R$ and let $N=\mathfrak{a} M$ and $\mathfrak{b}=\operatorname{Ann}(M / N)$. If $\mathfrak{b} \subseteq J(R), M$ is complete with respect to the 6 -adic topology, $\left\{P_{i}\right\}_{i \geq 1}$ is a countable family of prime submodules of $M$, and $x \in M$, then $x+N \subseteq \bigcup_{i \geq 1} P_{i}$ implies that $x+N \subseteq P_{j}$ for some $j \geq 1$. This extends a theorem of Sharp and Vámos concerning prime ideals to prime submodules.
\end{abstract}

2000 Mathematics Subject Classification. 13C99, 13J10, 13E05.

1. Introduction. Let $R$ be a commutative ring with identity. One of the fundamental cornerstones of commutative ring theory is the "prime avoidance" theorem, which states that, if $\mathfrak{p}_{1}, \ldots, \mathfrak{p}_{n}$ are prime ideals of $R$ and $\mathfrak{a}$ is an ideal of $R$ such that $\mathfrak{a} \subseteq \bigcup_{i=1}^{n} \mathfrak{p}_{i}$, then $\mathfrak{a} \subseteq \mathfrak{p}_{j}$ for some $1 \leq j \leq n$. In [4], the authors proved and used an extension of the following.

THEOREM 1.1. Let $R$ be a Noetherian local ring having maximal ideal $\mathrm{m}$. Let $\left\{\mathfrak{p}_{i}\right\}_{i \geq 1}$ be a countable family of prime ideals of $R, \mathfrak{a}$ an ideal of $R$, and $x \in R$. If $R$ is complete with respect to the $\mathfrak{m}$-adic topology, then $x+\mathfrak{a} \subseteq \bigcup_{i \geq 1} \mathfrak{p}_{i}$ implies that $x+\mathfrak{a} \subseteq \mathfrak{p}_{j}$ for some $j \geq 1$.

Consider $M$ as a unitary left $R$-module. A submodule $P$ of $M$ is called prime submodule, if $P \neq M$ and for each $r \in R$ and $m \in M, r m \in P$ implies that $r \in \operatorname{Ann}(M / P)$ or $m \in P$. Of course, if we regard $R$ as an $R$-module, then the concept of prime submodules is equivalent to the concept of prime ideals. Now it is natural to ask if the "prime avoidance" theorem is true for prime submodules. In fact the answer is affirmative as has been shown by Lu [2].

THEOREM 1.2. Let $M$ be an $R$-module and $P_{1}, \ldots, P_{n}$ be prime submodules of $M$. Let $N$ be a submodule of $M$ such that $N \subseteq \bigcup_{i=1}^{n} P_{i}$. Also assume that $\operatorname{Ann}\left(M / P_{j}\right) \nsubseteq \operatorname{Ann}\left(M / P_{k}\right)$ for $j \neq k$. Then $N \subseteq P_{j}$ for some $1 \leq j \leq n$.

The main purpose of this paper is to generalize the above theorem to a countable family of prime submodules.

2. Main result. Let $R$ be a Noetherian ring, $\mathfrak{a}$ an ideal of $R$ contained in the Jacobson radical of $R$, and $M$ a finitely-generated $R$-module. The $\mathfrak{a}$-adic topology is metrizable and one suitable metric $d$ may be given by requiring that, for $x, y \in M$ with $x$ and $y$ different, $d(x, y)=1 / 2^{t}$, where $t$ is the greatest integer $i$ such that $x-y \in \mathfrak{a}^{i} M$. 
Now, we apply the Baire's category theorem for deducing our main result. Note that the Baire's category theorem states that a complete metric space is not the union of a countable family of nowhere dense subsets, and a subset of a metric space $M$ is nowhere dense in $M$ if and only if its closure has no interior point (see [1, page 38]). In the sequel, we denote the Jacobson radical of $R$ by $J(R)$.

MAIN THEOREM 2.1. Let $M$ be a finitely-generated module over a Noetherian ring $R$. Suppose $\mathfrak{a}$ is an ideal of $R$ and let $N=\mathfrak{a} M$ and $\mathfrak{b}=\operatorname{Ann}(M / N)$. If $\mathfrak{b} \subseteq J(R), M$ is complete with respect to the 6 -adic topology, $\left\{P_{i}\right\}_{i \geq 1}$ is a countable family of prime submodules of $M$, and $x \in M$, then $x+N \subseteq \bigcup_{i \geq 1} P_{i}$ implies that $x+N \subseteq P_{j}$ for some $j \geq 1$.

We will need the following well-known result which is essentially due to Krull.

LEMMA 2.2. If $M$ is a finitely-generated module over a Noetherian ring $R$ and 6 an ideal of $R$ such that $\mathfrak{b} \subseteq J(R)$, then each submodule of $M$ with respect to the $b$-adic topology is closed (see [3, Theorem 8.10]).

Proof OF THE MAIN THEOREM. By Lemma 2.2, each submodule of $M$ and in particular $N$ is closed with respect to the $b$-adic topology. One easily obtains that $x+N$ is also a closed subset of $M$. So the completeness of $M$ yields that $x+N$ is a complete metric space. But by hypothesis we have $x+N=\bigcup_{i \geq 1}\left((x+N) \cap P_{i}\right)$ and therefore it turns out by Baire's category theorem that, there is some $j \geq 1$ for which the subset $(x+N) \cap P_{j}$ of $x+N$ is not nowhere dense. But $(x+N) \cap P_{j}$ is closed in $x+N$ and therefore the interior of $(x+N) \cap P_{j}$ in $x+N$ is not empty. Let $c$ be an element of the interior of $(x+N) \cap P_{j}$. Thus there exists an open subset $U$ of $M$ such that $c \in(x+N) \cap U \subseteq(x+N) \cap P_{j}$. But $c \in U$ implies that, there is $k \in \mathbb{N}$ such that $c+\mathfrak{b}^{k} M \subseteq U$ and hence $c \in(x+N) \cap\left(c+\mathfrak{b}^{k} M\right) \subseteq(x+N) \cap P_{j}$. It is easily deduced that $N \cap \mathfrak{b}^{k} M \subseteq P_{j}$. But $\mathfrak{b}^{k} M \subseteq N$, so $\mathfrak{b}^{k} M \subseteq P_{j}$ and $\mathfrak{b}^{k} \subseteq \operatorname{Ann}\left(M / P_{j}\right)$. Since $P_{j}$ is a prime submodule of $M$, it follows that $\operatorname{Ann}\left(M / P_{j}\right)$ is a prime ideal of $R$ and so $\mathfrak{b} \subseteq \operatorname{Ann}\left(M / P_{j}\right)$. Now we have $\mathfrak{a} \subseteq \mathfrak{b} \subseteq \operatorname{Ann}\left(M / P_{j}\right)$, and hence $\mathfrak{a} M=N \subseteq P_{j}$. But $x-c \in N$, so $x+N \subseteq P_{j}$, as required.

REMARK 2.3. (i) Note that if $M$ is a finitely-generated module over a Noetherian ring $R, \mathfrak{a}_{1} \subseteq \mathfrak{a}_{2}$ ideals of $R$, and $M$ complete with respect to the $\mathfrak{a}_{2}$-adic topology, then it is also complete with respect to the $\mathfrak{a}_{1}$-adic topology. This shows that, the main theorem is also true if we assume that $M$ is complete in $J(R)$-adic topology.

(ii) In the view of (i), it is clear that Theorem 1.1 is a particular case of the main theorem.

ACKNOWLEDGEMENT. This research was in part supported by a grant from Institute for Studies in Theoretical Physics and Mathematics (IPM).

\section{REFERENCES}

[1] E. T. Copson, Metric Spaces, Cambridge Tracts in Mathematics and Mathematical Physics, no. 57, Cambridge University Press, London, 1968. MR 37\#877. Zbl 177.25303.

[2] C.-P. Lu, Unions of prime submodules, Houston J. Math. 23 (1997), no. 2, 203-213. MR 2000c:13015. Zbl 885.13004. 
[3] H. Matsumura, Commutative Ring Theory, Cambridge Studies in Advanced Mathematics, vol. 8, Cambridge University Press, Cambridge, 1989. MR 90i:13001. Zbl 666.13002.

[4] R. Y. Sharp and P. Vámos, Baire's category theorem and prime avoidance in complete local rings, Arch. Math. (Basel) 44 (1985), no. 3, 243-248. MR 86h:13004. Zbl 546.13002.

M. R. Pournaki: Department of Mathematical Sciences, Sharif University of TeChNOLOGY, P.O. BOX 11365-9415, TEHRAN, IRAN AND SCHOOL OF MATHEMATICS, INSTITUTE FOR Studies in Theoretical Physics AND MAthematics, P.O. Box 19395-5746, TeHran, Iran

E-mail address: pournaki@karun. ipm.ac. ir

M. Tousi: Department of Mathematics, Shahid Beheshti University, Evin, Tehran 19834, IRAN AND SCHOOL OF MATHEMATICS, INSTITUTE FOR STUDIES IN THEORETICAL PHYSICS AND MATHEMATics, P.O. Box 19395-5746, TEHRAN, IRAN

E-mail address: mtousi@vax.ipm.ac.ir 


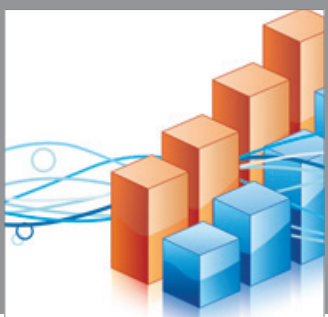

Advances in

Operations Research

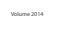

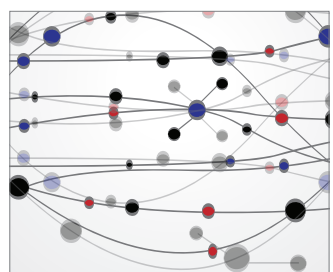

\section{The Scientific} World Journal
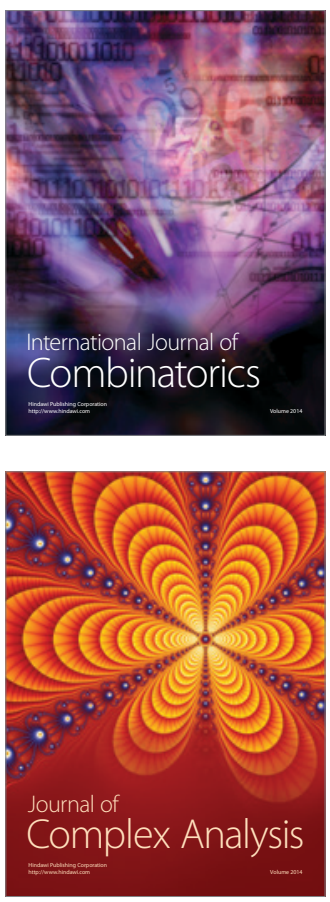

International Journal of

Mathematics and

Mathematical

Sciences
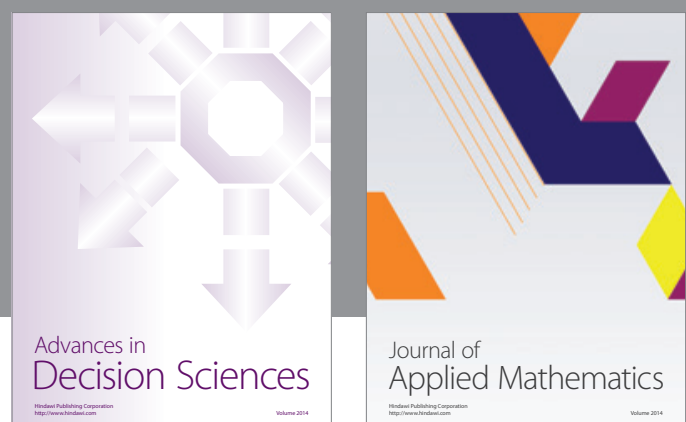

Journal of

Applied Mathematics
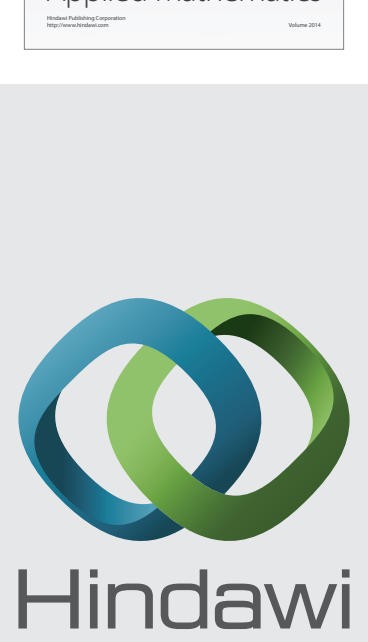

Submit your manuscripts at http://www.hindawi.com
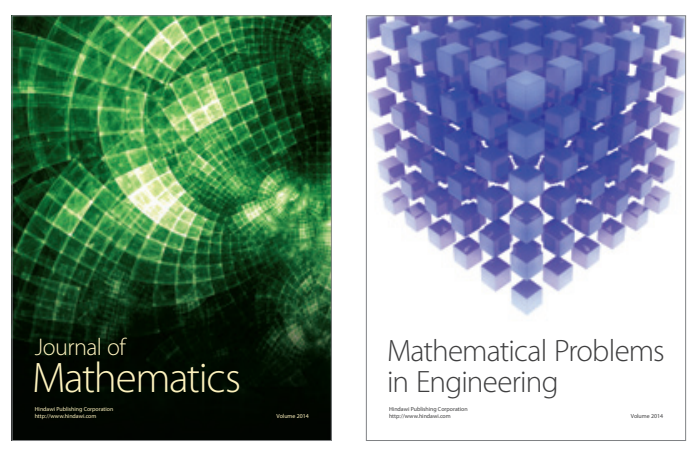

Mathematical Problems in Engineering
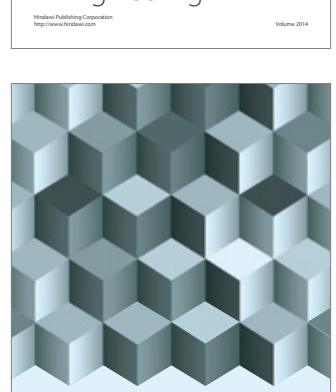

Journal of

Function Spaces
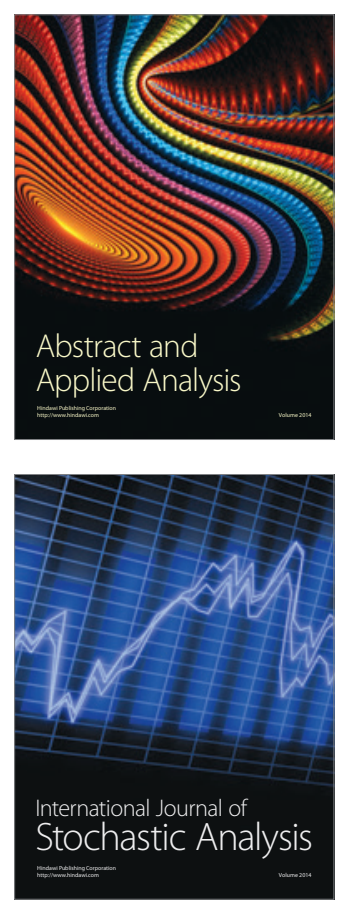

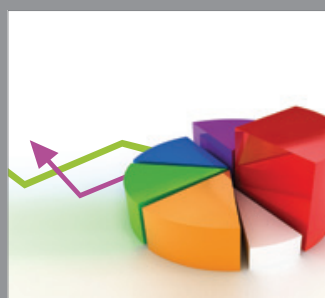

ournal of

Probability and Statistics

Promensencen
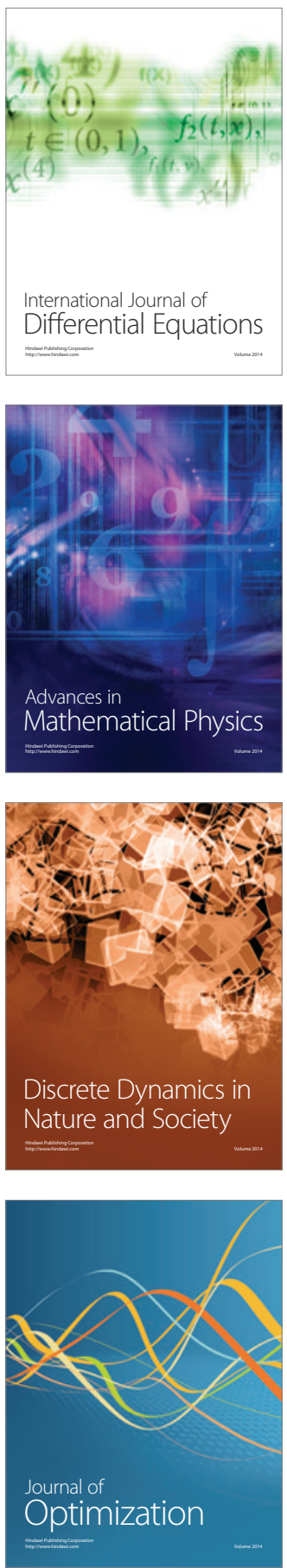